\title{
Iterative projection approach for phase retrieval of semi-sparse wave field
}

\author{
Rong Fan ${ }^{1}$, Qun Wan ${ }^{*}$, Fei Wen², Hui Chen ${ }^{1}$ and Yipeng Liu ${ }^{3}$
}

\begin{abstract}
In the paper, we consider the problem of two-dimensional (2D) phase retrieval, which recovers a 2D complex-valued wave field from magnitudes of both wave field and its Fourier transform. Due to the absence of the phase measurements, prior information on wave field is needed in order to recover phase, which is feasible when the phases of the wave field are sparse. In this paper, we improve the phase retrieval accuracy by incorporating phase sparse constraint of wave field. As a sequel to previous iterative projection approaches, iterative projection approaches with phase sparse constraint are realized based on 'soft thresholding'. It has superior performances in terms of convergence, residual error, noise stability, and suitability in large-scale phase retrieval problems. Numerical experiments illustrate that the proposed approach outperforms existing iterative projection approaches.
\end{abstract}

Keywords: Sparse signal recovery; Sparse phase retrieval; Iterative projection; Semi-sparse wave field; 2D Fourier transform

\section{Introduction}

Recovery of a signal from the magnitudes of its Fourier transform, also known as phase retrieval, is of great interest in applications such as astronomy [1], optical phased array [2], adaptive optics [3], and signal processing [4-6]. Up to now, there are two main categories of phase retrieval approaches in existing approaches, namely, semi-definite programming-based (SDP-based) approaches [7-11] and iterative projection approaches (Fienup-type methods) [12-18]. SDP-based approaches are not suitable for largescale problems, so we do not discuss them in the paper. For iterative projection approaches, the first proposed algorithm is the Gerchberg and Saxton approach (GS) [12]. In 1982, Fienup systematized the earlier works and introduced some types of phase-retrieval approaches: error reduction (ER) and the hybrid input-output algorithm (HIO) [13]. Besides, a few of iterative projection variants have been proposed, such as solvent flipping algorithm (SF) [14], averaged successive reflection algorithm (ASR) [15], hybrid projection reflection algorithm (HPR) [16], and relaxed averaged alternating reflectors

*Correspondence: wanqun@uestc.edu.cn

1 School of Electronic Engineering, University of Electronic Science and Technology of China, No. 2006, Xiyuan Ave, West Hi-Tech Zone, Chengdu 611731, China

Full list of author information is available at the end of the article algorithm (RAAR) [17]. In 2007, a comprehensive description about iterative projection approaches was reviewed [18]. Despite tremendous progress, iterative projection approaches are known to suffer from convergence issues and often do not lead to correct recovery especially in onedimensional (1D) problems. Alternating projection phasing approaches are plagued by stagnation and spurious errors partly due to intrinsic non-uniqueness of the standard phase retrieval problem. Recently, many researchers exploit the sparsity property of a signal as a priori information to recover signal, and many sparse signal processing approaches are proposed. Interested authors are encouraged to read literatures [19-22]. Motivated by sparse signal processing communities, many phase retrieval approaches by incorporating sparsity property of wave field are discussed [23-30]. However, it is just assumed that the underling wave field is sparse in these literatures. If the phases of a wave field are sparse but the magnitudes of wave field are not [31] (it is called as semi-sparse wave field in the paper), as far as we know, existing phase retrieval approaches with sparse constraint cannot utilize a priori information to recover the sparse phase. This is the reason why we address the issue in the paper. Different from existing sparse phase retrieval approaches in [23-30], we propose an iterative projection approach with phase sparse constraint for semi-sparse

\section{望 Springer}

(c) 2014 Fan et al: licensee Springer. This is an Open Access article distributed under the terms of the Creative Commons

Attribution License (http://creativecommons.org/licenses/by/2.0), which permits unrestricted use, distribution, and reproduction in any medium, provided the original work is properly credited. 
wave field. As phase sparse constraint is exploited, the proposed approach has superior performances than the existing iterative projection approaches in terms of rapid convergence, smaller residual error, noise stability, and suitability in large-scale phase retrieval problems. They are the main contributions of the paper.

The organization of this paper is as follows. Section 2 contains the problem formulation. The proposed iterative projection approach with phase sparse constraint is presented in Section 3. We demonstrate the performances of the proposed phase retrieval approach by numerical experiments in Section 4. Finally, the conclusions of the paper are given in Section 5.

\section{Problem formulation}

We denote 2D complex-valued discrete wave field by $u(\xi, \eta)$, which is related to its discrete Fourier transform (DFT) $U(x, y)$, by

$$
\begin{aligned}
U(x, y) & =|U(x, y)| \circ \exp \{j \psi(x, y)\} \\
& =\operatorname{DFT}\{u\} \\
& =\frac{1}{M N} \sum_{\xi=0}^{M-1} \sum_{\eta=0}^{N-1} u(\xi, \eta) \exp \left\{-j 2 \pi\left(\frac{x \xi}{M}+\frac{y \eta}{N}\right)\right\}
\end{aligned}
$$

where

$$
(\xi, \eta) \in \Gamma \triangleq\{0,1, \cdots, M-1\} \times\{0,1, \cdots, N-1\}
$$

is a $2 \mathrm{D}$ spatial coordinate, and $(x, y) \in \Pi \triangleq\{0,1, \cdots, M-$ $1\} \times\{0,1, \cdots, N-1\}$ is a $2 \mathrm{D}$ spatial frequency coordinate. The symbol ' $o$ ' denotes the Hadamard product. Meanwhile, we rewrite the complex-valued wave field $u(\xi, \eta)$ in polar form, which yields

$$
u(\xi, \eta)=|u(\xi, \eta)| \circ \exp \{j \phi(\xi, \eta)\} .
$$

Moreover, we also present the inverse discrete Fourier transform (IDFT) as

$$
\begin{aligned}
u(\xi, \eta) & =|u(\xi, \eta)| \circ \exp \{j \phi(\xi, \eta)\} \\
& =\operatorname{IDFT}\{U\} \\
& =\sum_{x=0}^{M-1} \sum_{y=0}^{N-1} U(x, y) \exp \left\{j 2 \pi\left(\frac{x \xi}{M}+\frac{y \eta}{N}\right)\right\} .
\end{aligned}
$$

Phase retrieval task is to recover $\phi(\xi, \eta)$ from measurements of both $|U(x, y)|$ and $|u(\xi, \eta)|$. In the paper, we assume that the phases of wave field are sparse, but its magnitudes are not (i.e., semi-sparse wave field). As the prior knowledge about $\phi(\xi, \eta)$ is employed in phase retrieval, we can obtain an improved recovery quality. Clearly, from the mathematical point of view, recovering phases of wave field can be stated as an inverse problem. Hereafter, we call $|U(x, y)|$ and $|u(\xi, \eta)|$ as Fourier plane measurement and object plane measurement, respectively.

\section{Problem solution}

\subsection{Conventional iterative projection approaches}

To the best of our knowledge, GS is the first successful iterative projection approach for the phase retrieval problem. Even more important, the work on GS approach was continued and improved by many researchers. A few modified iterative projection approaches have now been proposed. These approaches try to find the intersection between two sets (i.e., $\Gamma$ and $\Pi$ in Section 2) [13-18]. Typically, those are the set of all the possible objects with a given $|U(x, y)|$ and the set of all the objects that are constrained within $\Gamma$. The search for the intersection is based on the information obtained by projecting the current estimate on the two sets. An error metric is obtained by evaluating the distance between the current estimate and a given set. The block diagram of the iterative projection approaches is illustrated in Figure 1.

Besides GS, there are a few of other iterative projection approaches, such as ER, HIO, SF, ASR, and RAAR [13-18]. All of them have the same block diagram as in Figure 1. In the next, we take GS approach for an example to describe the procedures of iterative projection approach, which are shown in details in Algorithm 1.

Repeat for $i=0,1,2, \cdots$

Step 1: $i$ th iterative wave field $u^{i}(\xi, \eta)=|u(\xi, \eta)| \circ \exp \left\{j \phi^{i}(\xi, \eta)\right\}$;

Step 2: Update of the wave field by the object plane measurement on set $\Gamma$, i.e., $u^{i}(\xi, \eta)=\left\{\begin{array}{cc}|u(\xi, \eta)| \circ \exp \left\{j \phi^{i}(\xi, \eta)\right\} & (\xi, \eta) \in \Gamma \\ 0 & \text { otherwise }\end{array}\right.$

Step 3: Forward Fourier transform, i.e., $U^{i}(x, y)=\operatorname{DFT}\left\{u^{i}\right\}=\left|U^{i}(x, y)\right| \circ \exp \left\{j \psi^{i}(x, y)\right\}$

Step 4: Update of the Fourier image by the Fourier plane measurement $|U(x, y)|$ on set $\Pi$, i.e.,

$U^{i}(x, y)=\left\{\begin{array}{cc}|U(x, y)| \circ \exp \left\{j \psi^{i}(x, y)\right\} & (x, y) \in \Pi \\ 0 & \text { otherwise }\end{array}\right.$

Step 5: Backward Fourier transform (inverse Fourier transform), i.e., $u^{i}(\xi, \eta)=\operatorname{IDFT}\left\{U^{i}\right\} \triangleq\left|u^{i}(\xi, \eta)\right| \circ \exp \left\{j \phi^{i}(\xi, \eta)\right\}$ and $\phi^{i} \leftarrow \mathbf{w} \circ \phi^{i}$, where $\mathbf{w}$ is a window function.

Step 6: If phase residual error $\sigma$ or a maximum number of iterations $I$ is reached, stop the procedure,

else return to Step 1. 


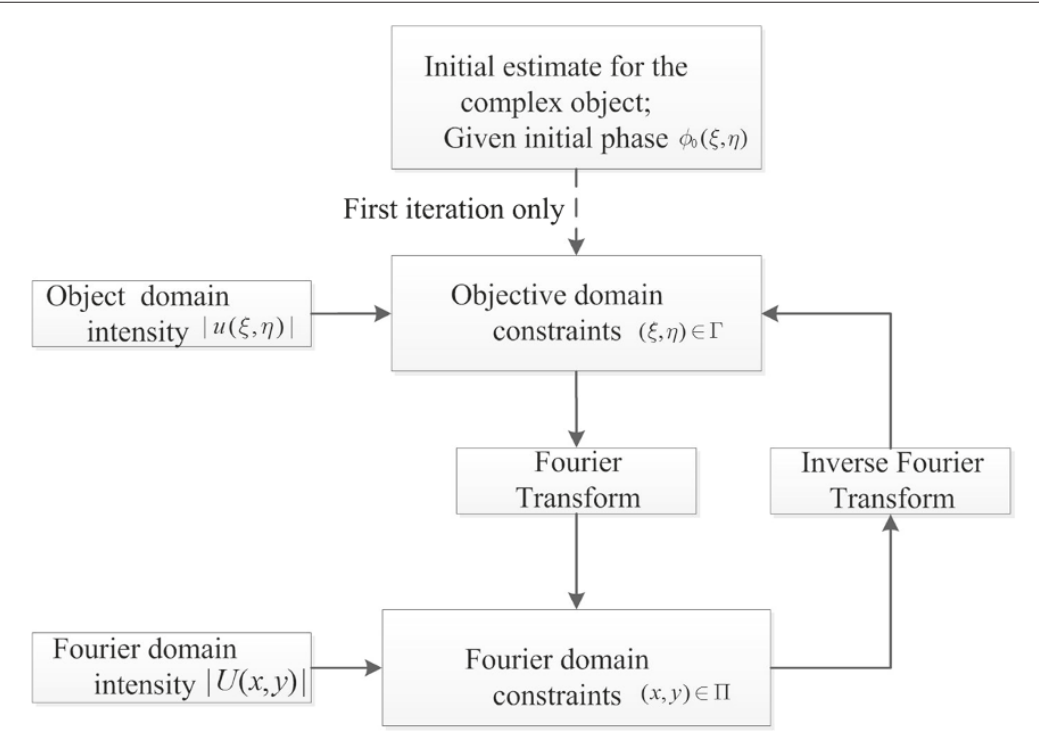

Figure 1 Block diagram of the iterative projection approaches.

Remark 1. Noted that the presented GS-type approach in Algorithm 1 is different from the standard GS approach because there is an additional window operator in step 5 . Although it is a simple modification based on standard GS approach by introducing window function, interestingly, simulation experiment shows that it does improve recovery performance in Section 4. For simplicity, we call the GS-type approach with window function as the GS approach in the paper.

Remark 2. The uniqueness of phase retrieval: In general, the unique phase retrieval from Fourier transform magnitude-only data is impossible. However, we utilize magnitudes of semi-sparse wave field and its Fourier transform; two measurements are used to resolve the phase ambiguity. To recover $M \times N$ phases, there are $2 M \times N$ unknown variables, and hence, we can resolve all unknown variables using two measurements. If the solution exists, it must be unique. This is the reason why there is no phase ambiguity problem in the paper.

Remark 3. Explanations of window operator: For the GS-type approach with window function in Algorithm 1, we present some trivial explanations why window operator $\mathbf{w}$ is helpful to improve the reconstruction performance. Because using window operator is actually equivalent to introduce some prior information for the phases of semi-sparse wave field, consider an extreme case, for example, if the window operator is the phase support of the semi-sparse wave field, the phase support of wave field is known exactly. Obviously, it improves the reconstruction performance from the aspect with window operator.

\subsection{The proposed iterative projection approaches with phase sparse constraint}

In subsection 3.1, we discussed some existing iterative projection approaches. However, all of them do not consider the structure property about $\phi(\xi, \eta)$. As a matter of fact, we can utilize this property to improve the recovery performance when $\phi(\xi, \eta)$ is sparse.

In this subsection, we present a counterpart of iterative projection approach with phase sparse constraint for phase retrieval. The approach starts with a random initialization of the phase in each iteration; the inverse Fourier transform is computed as the same as the conventional iterative projection approaches. The phase of inverse Fourier transform filtered by a 'soft thresholding' operator is then computed to obtain the redefined phase estimate. This procedure is repeated until phase residual error or a maximum number of iterations is reached.

Next, we propose a soft thresholding strategy to keep $\phi(\xi, \eta)$ be sparse in each iterative step. The soft thresholding operator is defined as

$$
\mathrm{TH}_{\lambda w}=\operatorname{sign}(w)(|w|-\lambda)^{+}, \text {where }(\cdot)^{+}=\max (\cdot, 0) .
$$

The thresholding parameter $\lambda$ makes a fixed portion of the elements of $\phi(\xi, \eta)$ be non-zero. As a matter of fact, the soft thresholding strategy is popular in many other iterative approaches such as alternative direction method (ADM) [5]. Similar to conventional iterative projection approaches, we present a block diagram of the iterative projection approaches with phase sparse constraint in Figure 2. 
In fact, it can be extended into a few of iterative projection approaches with phase sparse constraint. To distinguish from conventional ER, HIO, ASR, and RAAR, we denote by iterative projection approaches with phase sparse constraint ER-PhSparse, HIO-PhSparse, ASRPhSparse, RAAR-PhSparse, respectively. All of them have the same block diagram as in Figure 2. For simplicity, we take GS for example to describe the procedures of iterative projection approach with phase sparse constraint, which is shown in Algorithm 2.

\section{Algorithm 2 GS-type iterative projection approach with phase sparse constraint (GS-PhSparse) for phase retrieval}

Initialization: initial phase $\phi^{0}(\xi, \eta)$, object plane measurement $|u(\xi, \eta)|$ and Fourier plane measurement $|U(x, y)|$, spatial coordinate set $(\xi, \eta) \in \Gamma$ and spatial frequency coordinate set $(x, y) \in \Pi$, threshold parameter $\lambda$ and residual error $\sigma$, maximum number of iterations $I$ are given and set $i=0$;

Step 1: $i$ th iterative wave field

$u^{i}(\xi, \eta)=|u(\xi, \eta)| \circ \exp \left\{j \phi^{i}(\xi, \eta)\right\}$;

Step 2: Update of the wave field by the object plane measurement on set $\Gamma$, i.e.,

$u^{i}(\xi, \eta)=\left\{\begin{array}{cc}|u(\xi, \eta)| \circ \exp \left\{j \phi^{i}(\xi, \eta)\right\} & (\xi, \eta) \in \Gamma \\ 0 & \text { otherwise }\end{array}\right.$

Step 3: Forward Fourier transform, i.e.,

$U^{i}(x, y)=\operatorname{DFT}\left\{u^{i}\right\}=\left|U^{i}(x, y)\right| \circ \exp \left\{j \psi^{i}(x, y)\right\}$

Step 4: Update of the Fourier image by the Fourier plane measurement $|U(x, y)|$ on set $\Pi$, i.e.,

$U_{t}^{i}(x, y)=\left\{\begin{array}{cc}|U(x, y)| \circ \exp \left\{j \psi^{i}(x, y)\right\} & (x, y) \in \Pi \\ 0 & \text { otherwise }\end{array}\right.$

Step 5: Backward Fourier transform (inverse Fourier transform), i.e.,

$u_{t}^{i}(\xi, \eta)=\operatorname{IDFT}\left\{U_{t}^{i}\right\} \triangleq\left|u_{t}^{i}(\xi, \eta)\right| \circ \exp \left\{j \phi_{t}^{i}(\xi, \eta)\right\}$ and

$\phi_{t}^{i} \leftarrow \mathbf{w} \circ \phi_{t}^{i}$, where $\mathbf{w}$ is a window function.

Step 6: Update phase of the complex-valued signal,

$\phi^{i+1}(\xi, \eta) \leftarrow \mathrm{TH}_{\lambda}\left\{\phi_{t}^{i}(\xi, \eta)\right\}=\operatorname{sign}\left(\phi_{t}^{i}(\xi, \eta)\right)\left(\left|\phi_{t}^{i}(\xi, \eta)\right|-\lambda\right)^{+}$ where, $(\cdot)^{+}=\max (\cdot, 0)$

Step 7: If phase residual error $\sigma$ or a maximum number of iterations $I$ is reached, stop the procedure, else $i \leftarrow i+1$ and return to Step 1.

\subsubsection{Parameter $\lambda$ selection}

It is noted that the thresholding parameter $\lambda$ in Algorithm 2 is in the range $[0,1]$. In order to select the parameter $\lambda$ properly, in the paper, we select different $\lambda$ values within the range $[0,1]$ in ascending order and denote them by $\left\{\lambda_{1}, \lambda_{2}, \cdots, \lambda_{P}\right\}$ at first. And then, by given $\lambda_{i}$, we calculate relative error $e(i)=\left\|\phi_{\lambda_{i}}(\psi, \eta)-\phi_{\lambda_{i+1}}(\psi, \eta)\right\|_{F}$ with the procedures in Algorithm 2, where $\phi_{\lambda_{i}}(\psi, \eta)$ is the recovered phase given by $\lambda_{i}, i=1,2, \cdots, P$. Finally, we fit a second-order polynomial function $\operatorname{err}(\lambda)$ with $\left(\lambda_{i}, e(i)\right)$ for $i=1,2, \cdots, P$, and we select $\lambda^{*}=\operatorname{argmin} \operatorname{err}(\lambda)$ as the optimal value.

The convergence of the proposed GS-PhSparse approach cannot be guaranteed. It remains to be a difficult problem in the phase retrieval. A mathematical rigorous analysis has not been found in many existing approaches for conventional phase retrieval including ER, HIO, ASR, and RAAR. However, we must point out that the error reduction holds in the GS-PhSparse approach. The main result of error reduction of the GS-PhSparse is presented in Theorem 1 .

Theorem 1. For the ith iteration, define the mean squared error (MSE) as

$$
E_{i} \triangleq \frac{1}{M N} \sum_{x=0}^{M-1} \sum_{y=0}^{N-1}\left[\left|U^{i}(x, y)\right|-|U(x, y)|\right]^{2},
$$

where $\left|U^{i}(x, y)\right|$ and $|U(x, y)|$ are Fourier transform magnitude of $u^{i}(x, y)$ and Fourier domain measured magnitude, respectively. $u^{i}(\xi, \eta)$ is the recovered signal in ith iteration. $u_{t}^{i}(\xi, \eta)$ is defined the same as in step 5 in Algorithm 2. For $\forall \xi, \forall \eta$, if

$$
\begin{gathered}
\operatorname{sign}\left(\frac{u^{i}(\xi, \eta)+u^{i+1}(\xi, \eta)}{2}-u_{t}^{i}(\xi, \eta)\right) \\
=\operatorname{sign}\left(u^{i}(\xi, \eta)-u^{i+1}(\xi, \eta)\right)
\end{gathered}
$$

holds, then the error in $(i+1)$ th iteration is less than or equals that in the ith iteration, i.e., $E_{i} \geqslant E_{i+1}$, for $\forall i$.

Proof 1. To consider

$$
\mathrm{E}_{i}=\frac{1}{M N} \sum_{x=0}^{M-1} \sum_{y=0}^{N-1}\left[\left|U^{i}(x, y)\right|-|U(x, y)|\right]^{2} .
$$

Since $\left|U_{t}^{i}(x, y)\right|=|U(x, y)|$, for $\forall i$, yields,

$$
\mathrm{E}_{i}=\frac{1}{M N} \sum_{x=0}^{M-1} \sum_{y=0}^{N-1}\left[\left|U^{i}(x, y)\right|-\left|U_{t}^{i}(x, y)\right|\right]^{2},
$$

where $|U(x, y)|$ is the observed Fourier magnitude. It is noted that $\left|U^{i}(x, y)\right|$ and $\left|U_{t}^{i}(x, y)\right|$ have the same phase $\psi^{i}(x, y)$, and hence, we can rewrite that

$$
\begin{aligned}
\mathrm{E}_{i} & =\frac{1}{M N} \sum_{x=0}^{M-1} \sum_{y=0}^{N-1}\left[\left|U^{i}(x, y)\right|-\left|U_{t}^{i}(x, y)\right|\right]^{2} \\
& =\frac{1}{M N} \sum_{x=0}^{M-1} \sum_{y=0}^{N-1}\left|U^{i}(x, y)-U_{t}^{i}(x, y)\right|^{2} \\
& =\sum_{\xi=0}^{M-1} \sum_{\eta=0}^{N-1}\left|u^{i}(\xi, \eta)-u_{t}^{i}(\xi, \eta)\right|^{2},
\end{aligned}
$$




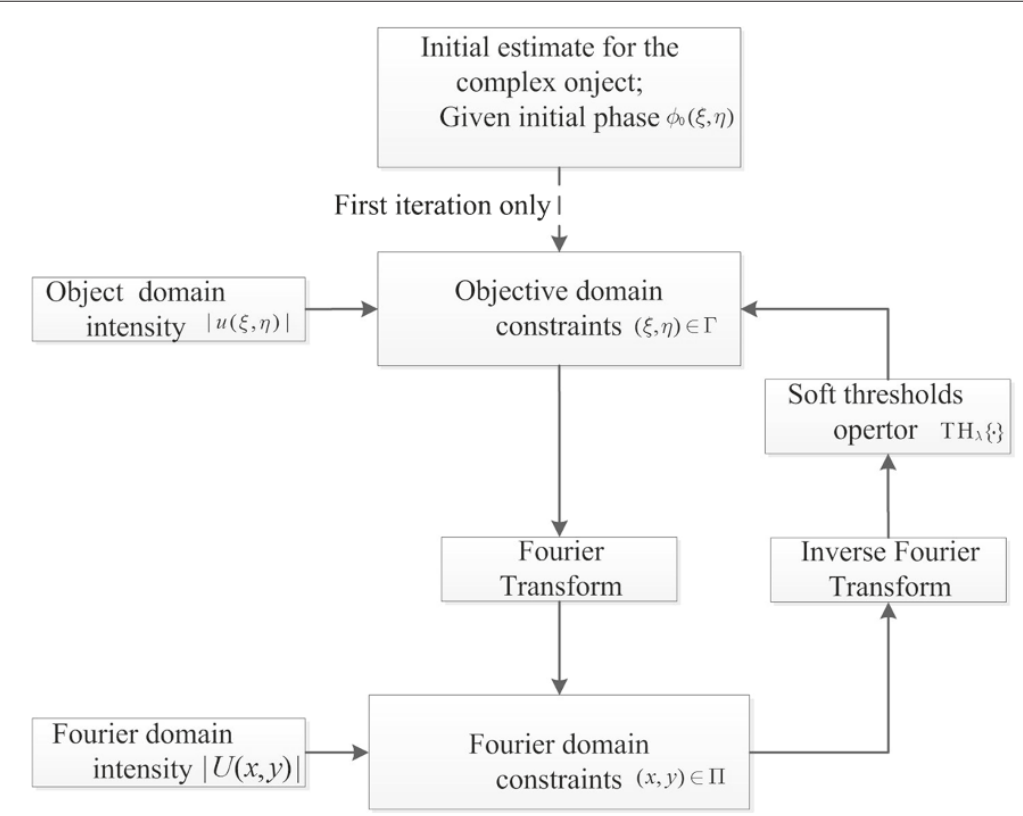

Figure 2 Block diagram of the iterative projection approaches with phase sparse constraint.

where the last equality above follows Parseval's theorem. Meanwhile, we also have a relationship as following:

$$
\begin{aligned}
& \sum_{\xi=0}^{M-1} \sum_{\eta=0}^{N-1}\left|u^{i+1}(\xi, \eta)-u_{t}^{i}(\xi, \eta)\right|^{2} \\
& \quad=\frac{1}{M N} \sum_{x=0}^{M-1} \sum_{y=0}^{N-1}\left|U^{i+1}(x, y)-U_{t}^{i}(x, y)\right|^{2} \\
& \geqslant \frac{1}{M N} \sum_{x=0}^{M-1} \sum_{y=0}^{N-1}\left[\left|U^{i+1}(x, y)\right|-\left|U_{t}^{i}(x, y)\right|\right]^{2} \\
& \quad=\frac{1}{M N} \sum_{x=0}^{M-1} \sum_{y=0}^{N-1}\left[\left|U^{i+1}(x, y)\right|-|U(x, y)|\right]^{2} \\
& =\mathrm{E}_{i+1},
\end{aligned}
$$

where the above inequality follows as a direct consequence of the triangle inequality. On the other hand, since $\operatorname{sign}\left(\frac{u^{i}(\xi, \eta)+u^{i+1}(\xi, \eta)}{2}-u_{t}^{i}(\xi, \eta)\right)=\operatorname{sign}\left(u^{i}(\xi, \eta)-\right.$ $\left.u^{i+1}(\xi, \eta)\right)$ for $\forall \xi, \forall \eta$, and we have

Case 1. if $\frac{u^{i}(\xi, \eta)+u^{i+1}(\xi, \eta)}{2} \leqslant u_{t}^{i}(\xi, \eta)$, and $u^{i}(\xi, \eta) \leqslant$ $u^{i+1}(\xi, \eta)$, we can get

$$
\begin{aligned}
\left(\left(u^{i}(\xi, \eta)\right.\right. & \left.+u^{i+1}(\xi, \eta)\right) \cdot\left(u^{i}(\xi, \eta)-u^{i+1}(\xi, \eta)\right) \\
& \geqslant 2 u_{t}^{i}(\xi, \eta) \cdot\left(u^{i}(\xi, \eta)-u^{i+1}(\xi, \eta)\right)
\end{aligned}
$$

for $\forall \xi, \forall \eta$, equivalently,

$$
\begin{aligned}
\left|u^{i}(\xi, \eta)\right|^{2} & -2 u_{t}^{i}(\xi, \eta) u^{i}(\xi, \eta)+\left|u_{t}^{i}(\xi, \eta)\right|^{2} \geqslant\left|u^{i+1}(\xi, \eta)\right|^{2} \\
& -2 u_{t}^{i}(\xi, \eta) \cdot u^{i+1}(\xi, \eta)+\left|u_{t}^{i}(\xi, \eta)\right|^{2}
\end{aligned}
$$

for $\forall \xi, \forall \eta$, yields

$$
\begin{aligned}
& \sum_{\xi=0}^{M-1} \sum_{\eta=0}^{N-1}\left|u^{i}(\xi, \eta)-u_{t}^{i}(\xi, \eta)\right|^{2} \\
& \geqslant \sum_{\xi=0}^{M-1} \sum_{\eta=0}^{N-1}\left|u^{i+1}(\xi, \eta)-u_{t}^{i}(\xi, \eta)\right|^{2}
\end{aligned}
$$

Case 2. If $\frac{u^{i}(\xi, \eta)+u^{i+1}(\xi, \eta)}{2} \geqslant u_{t}^{i}(\xi, \eta)$ and $u^{i}(\xi, \eta) \geqslant$ $u^{i+1}(\xi, \eta)$, in the same way, we can also have

$$
\begin{aligned}
& \sum_{\xi=0}^{M-1} \sum_{\eta=0}^{N-1}\left|u^{i}(\xi, \eta)-u_{t}^{i}(\xi, \eta)\right|^{2} \\
& \geqslant \sum_{\xi=0}^{M-1} \sum_{\eta=0}^{N-1}\left|u^{i+1}(\xi, \eta)-u_{t}^{i}(\xi, \eta)\right|^{2}
\end{aligned}
$$

Combined with (9) and (10), we know that $E_{i} \geqslant E_{i+1}$ holds for $\forall i$, immediately.

Generally speaking, it is hard to prove whether Equation (6) holds or not with mathematical analysis. However, the above theorem presents a sufficient, but not a necessary condition which ensures that the MSE decreases. It is very 
important in theory. As a matter of fact, the inequality relationship (14) holds for general semi-sparse wave field, which is confirmed in Section 4 by numerical simulations. In the next, we present another meaningful result, which tells us that the recovered phase error is bounded with the proposed approach.

Theorem 2. Let $\phi^{i}(\xi, \eta)$ be the recovered phase of signals in the ith iterative signal. $\phi_{t}^{i}(\xi, \eta)$ is an inverse Fourier transform of $U_{t}^{i}(x, y)$, which is defined in step 5 of Algorithm 2. $\phi^{i+1}(\xi, \eta)$ is the updated phase of the semi-sparse wave field in the ith iteration. If phase $\phi(\xi, \eta)$ is a K-sparse on support set $S$, namely, $S \triangleq\{\phi(\xi, \eta):|\operatorname{supp}\{\phi(\xi, \eta)\}|=$ $K\}$, then there exists a thresholding parameter $\lambda$, with the phase update rule $\phi^{i+1}(\xi, \eta) \leftarrow T H_{\lambda}\left\{\phi_{t}^{i}(\xi, \eta)\right\}=$ sign $\left(\phi_{t}^{i}(\xi, \eta)\right)\left(\left|\phi_{t}^{i}(\xi, \eta)\right|-\lambda\right)^{+}$, the recovered relative phase residual $\|\hat{\phi}(\xi, \eta)-\phi(\xi, \eta)\|_{F} /\|\phi(\xi, \eta)\|_{F}$ is bounded.

Proof 2. Consider $\phi^{i+1}(\xi, \eta)$ is the best possible sparse approximation with $K$-sparse (because it is formed by retaining the $K$ largest entries in $\phi_{t}^{i}(\xi, \eta)$ and setting others to zero) and $\phi^{i}(\xi, \eta)$ is also $K$-sparse. And, thus, we have

$$
\begin{aligned}
\phi^{i+1}(\xi, \eta) & =\arg \min _{\phi(\xi, \eta) \in S}\left\|\phi(\xi, \eta)-\phi_{t}^{i}(\xi, \eta)\right\|_{F}^{2} \\
& =\arg \min _{\phi(\xi, \eta) \in S} \sum_{\xi=0}^{M-1} \sum_{\eta=0}^{N-1}\left|\phi(\xi, \eta)-\phi_{t}^{i}(\xi, \eta)\right|^{2} .
\end{aligned}
$$

Since $\phi^{i}(\xi, \eta) \in S$, we have the inequality

$$
\begin{aligned}
& \sum_{\xi=0}^{M-1} \sum_{\eta=0}^{N-1}\left|\phi^{i}(\xi, \eta)-\phi_{t}^{i}(\xi, \eta)\right|^{2} \\
& \geqslant \sum_{\xi=0}^{M-1} \sum_{\eta=0}^{N-1}\left|\phi^{i+1}(\xi, \eta)-\phi_{t}^{i}(\xi, \eta)\right|^{2} .
\end{aligned}
$$

Therefore, the phase residual in the $(i+1)$ th iteration is less than or equals that in the $i$ th iteration. In the above inequality, the equality holds if and only if $\phi^{i}(\xi, \eta)=\phi^{i+1}(\xi, \eta)$. It shows that the recovered phase residual is bounded, since there exists a one-to-one mapping between sparsity degree and thresholding parameter $\lambda$. In other words, if $|\operatorname{supp}\{\phi(\xi, \eta)\}|=K$, there must exist $\lambda$ and make the non-zero number of entries in $\mathrm{TH}_{\lambda}\left\{\phi_{t}^{i}(\xi, \eta)\right\}$ equal to $K$. Therefore, using the phase update rule in GS-PhSparse, the recovered phase residual $\|\hat{\phi}(\xi, \eta)-\phi(\xi, \eta)\|_{F}$ is bounded. Certainly, $\| \hat{\phi}(\xi, \eta)-$ $\phi(\xi, \eta)\left\|_{F} /\right\| \phi(\xi, \eta) \|_{F}$ is also bounded.

Once again, we point out that the corresponding iterative projection approaches with phase sparse constraint (i.e., ER-PhSparse, HIO-PhSparse, ASR-PhSparse, and
RAAR-PhSparse) can be derived directly. Considering GS is the first successful iterative projection approach for phase retrieval and RAAR is the latest proposed version, in the next section, we just employ GS and GS-PhSparse, RAAR, and RAAR-PhSparse for comparison.

\section{Numerical simulations}

In this section, we provide several numerical simulations to demonstrate the performance of the proposed approach. Two images, 'Lena' and 'phantom', are taken to imitate the magnitude distribution and sparse phase distribution of wave field $u(\xi, \eta)$, respectively. The wave field size is $256 \times 256$. Magnitudes of wave field are nonnegative and its phases are sparse. The magnitude distribution and phase distribution of wave field $u(\xi, \eta)$ are shown in Figure $3 a, b, c$, respectively. Figure $3 c$ is obtained by setting all elements which are greater than 0.3 in Figure $3 b$ to 0 . The range of true phases in Figure $3 \mathrm{c}$ is $[0, \pi / 2]$. To estimate the recovery, we define normalized residual as

$$
\text { R_Error }=\frac{\|\hat{\phi}(\xi, \eta)-\phi(\xi, \eta)\|_{F}}{\|\phi(\xi, \eta)\|_{F}} \text {. }
$$

The approach is terminated if R_Error $\leqslant 10^{-4}$ or a maximum number of iterations, 100 , is reached. The

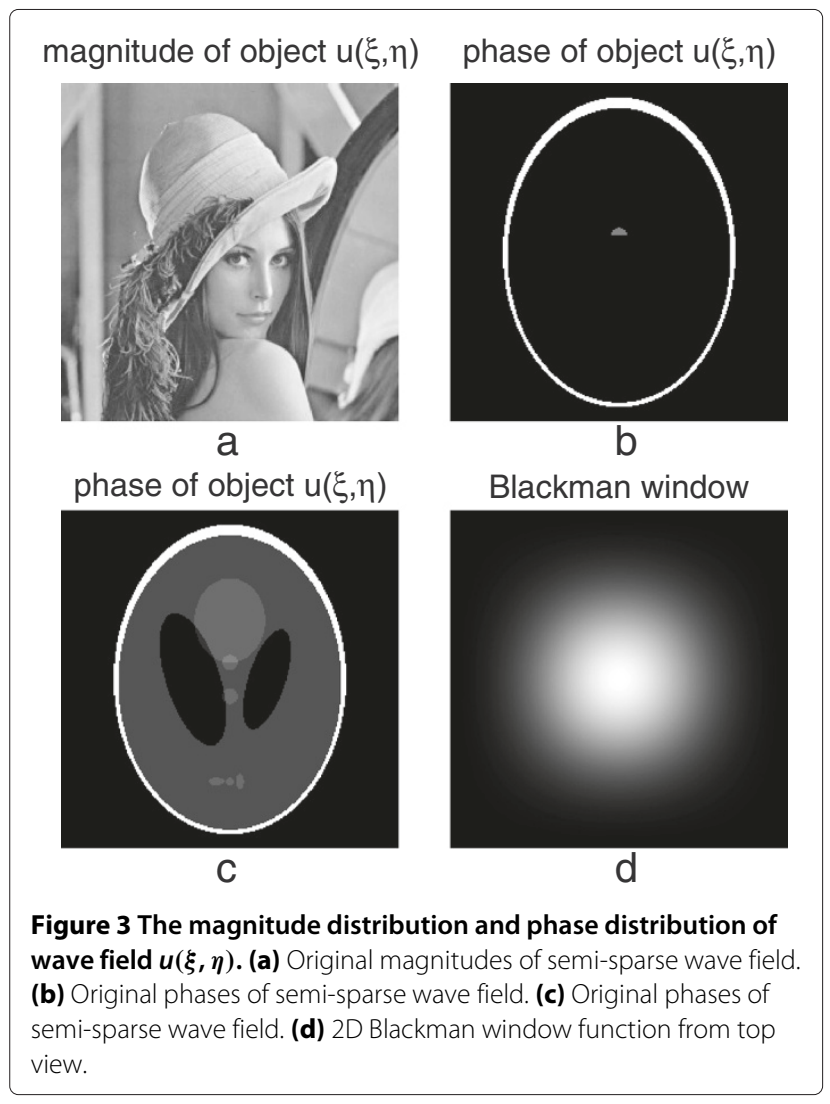


reconstruction accuracy is characterized by relative signal-to-noise ratio (RSNR), which is defined as

$$
\mathrm{RSNR}=-20 \log 10\left(\frac{\|\hat{\phi}(\xi, \eta)-\phi(\xi, \eta)\|_{F}}{\|\phi(\xi, \eta)\|_{F}}\right)
$$

Under these assumptions, we do several experiments from several aspects below.

Experiment 1. In order to illustrate that the recovery performance can be improved with window function, in this experiment, we calculate the RSNR with Blackman window function and rectangle window function. The Blackman window function and rectangle window function are presented in the following: The Blackman window of length $N$ is defined as $\mathbf{w}=w_{\text {blackman }} \cdot w_{\text {blackman }}^{T}$, and $w_{\text {blackman }}(n)=0.42-0.5 \cos (2 \pi n /(N-1))+0.08$ $\cos (4 \pi n /(N-1))$ for $0 \leqslant n \leqslant M-1$, where $M$ is $N / 2$ for even $N$ and $(N+1) / 2$ for odd $N$. The window function from the top view is shown in Figure 3d. In this experiment, we use Figure $3 a, b$ to imitate the magnitude distribution and sparse phase distribution of $u(\xi, \eta)$, respectively.

Assume that object plane measurement and the Fourier plane measurement are contaminated by Gaussian noise with SNR $=30 \mathrm{~dB}$. The simulation result is shown in Figure 4. Alternatively, keeping the same noise level and changing the sparse phase of the wave field with Figure 3c, we also calculate the RSNR of the GS approach using different window functions. The simulation result is shown in Figure 5. According to Figures 4 and 5, the advantages with window function are obvious.

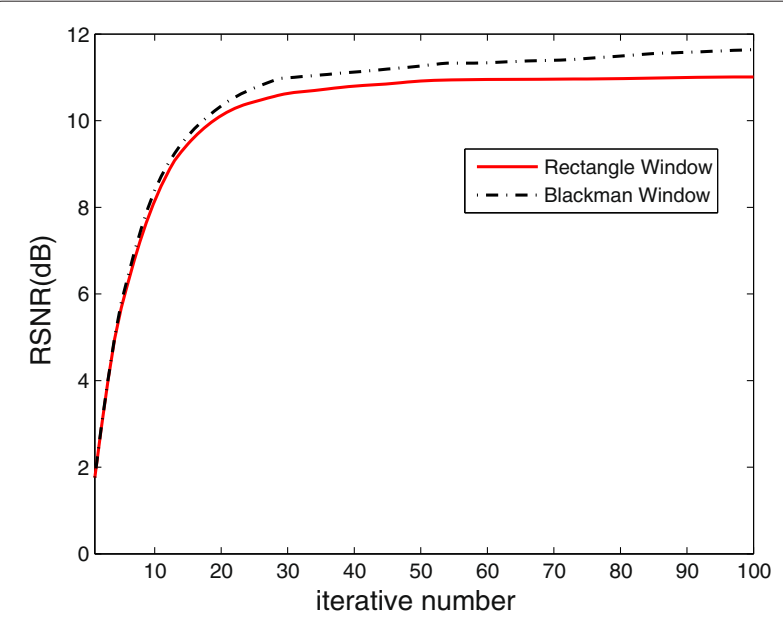

Figure 4 RSNR of Figure $3 a, b$ with standard GS approach and GS approach with window function.

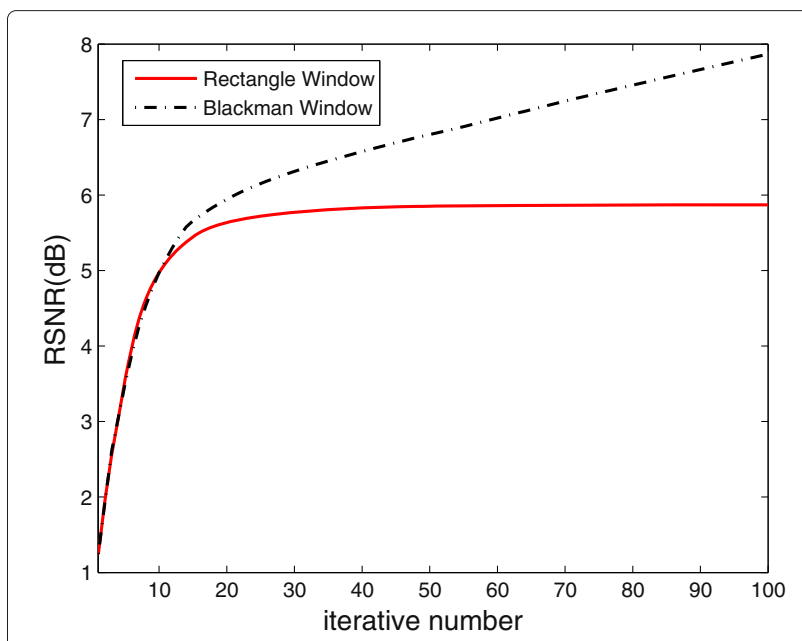

Figure 5 RSNR of Figure 3a,c with standard GS approach and GS approach with window function.

Experiment 2. As stated in Section 3, Theorem 1 tells us that the iterative residual decreases along with the increase of the iterative number, provided that Equation (6) holds. However, Equation (6) is a sufficient, but not a necessary condition, which decreases the MSE. It is not an easy problem to answer whether Equation (6) satisfies or not. As a matter of fact, if inequality (13) (certainly the same as (14)) is satisfied, then surely the MSE will decrease. Fortunately, this requirement is not too strict, which is confirmed by numerical simulation. In the experiment, we take Figure $3 \mathrm{a}, \mathrm{b}$ as magnitudes and phases of semi-sparse wave field, respectively. Object plane measurement and Fourier plane measurement are contaminated by Gaussian noise with SNR $=20 \mathrm{~dB}$. We calculate the iterative error between $\sum_{\xi=0}^{M-1} \sum_{\eta=0}^{N-1}\left|u^{i}(\xi, \eta)-u_{t}^{i}(\xi, \eta)\right|^{2}$ and $\sum_{\xi=0}^{M-1} \sum_{\eta=0}^{N-1}\left|u^{i+1}(\xi, \eta)-u_{t}^{i}(\xi, \eta)\right|^{2}$ with GS, GS-PhSparse, RAAR, and RAAR-PhSparse approaches, respectively. Especially, the iterative error with GS and GS-PhSparse is shown in Figure 6a, and the iterative error with RAAR and RAAR-PhSparse is shown in Figure 6b. The results of Figure 6 show that inequality (13) (or (14)) holds.

Experiment 3. Although it is difficult to set parameter $\lambda$, we present a practical method to select parameter $\lambda$ in subsection 3.2. In this experiment, we use the method proposed in subsection 3.2, which is required in the following experiments. For different noise levels, we evaluate $\left(e(i), \lambda_{i}\right)$ with the GS-PhSparse approach, where $\lambda_{i}=$ $\{0.01,0.11,0.22,0.33,0.44,0.55,0.66,0.77,0.88,0.99\}$, respectively. The normalized $e(i)$ versus $\lambda_{i}$ is plotted in Figure 7 in discrete black ' $x$ '. The fitted function $\operatorname{err}(\lambda)$ is 


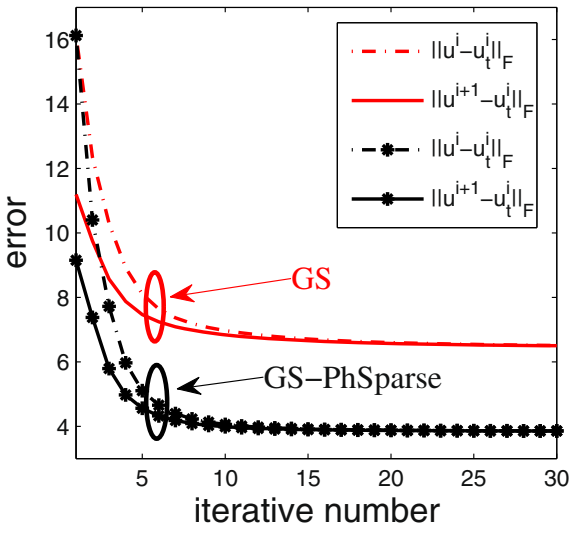

a

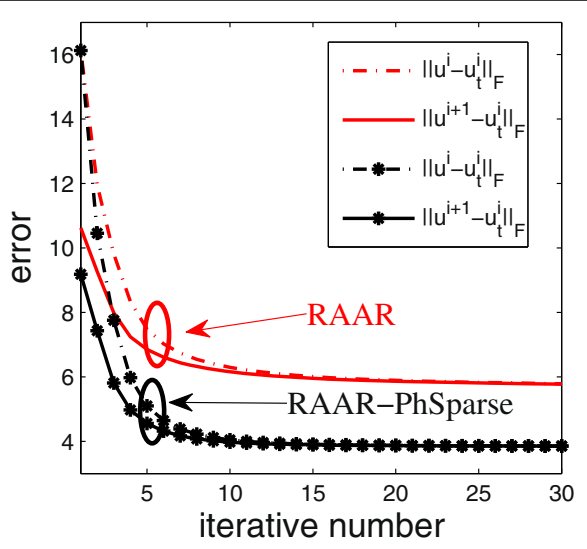

b

Figure 6 Iterative phase residual of semi-sparse wave field. (a) Iterative phase residual of semi-sparse wave field with GS and GS-PhSparse after 30 iterations. (b) Iterative phase residual of semi-sparse wave field with RAAR and RAAR-PhSparse after 30 iterations.

plotted in Figure 7 in red '-*' line. Specifically, Figure 7a is evaluated with $\mathrm{SNR}=10 \mathrm{~dB}$. Figure $7 \mathrm{~b}$ is evaluated with $\mathrm{SNR}=20 \mathrm{~dB}$. Figure $7 \mathrm{c}$ is evaluated with $\mathrm{SNR}=30 \mathrm{~dB}$, and Figure $7 \mathrm{~d}$ is evaluated with noiseless case. We can obtain similar results with the RAAR-PhSparse approach. Thus, we do not plot the results with the RAAR-PhSparse approach. According to Figure 7, we select thresholding parameter $\lambda=0.5$ properly.

Experiment 4. In the experiment, we compare two iterative projection approaches for phase retrieval: GS and the proposed iterative projection approach for phase retrieval with phase sparse constraint (i.e., GS-PhSparse). The approaches start with an all-zero initialization of the
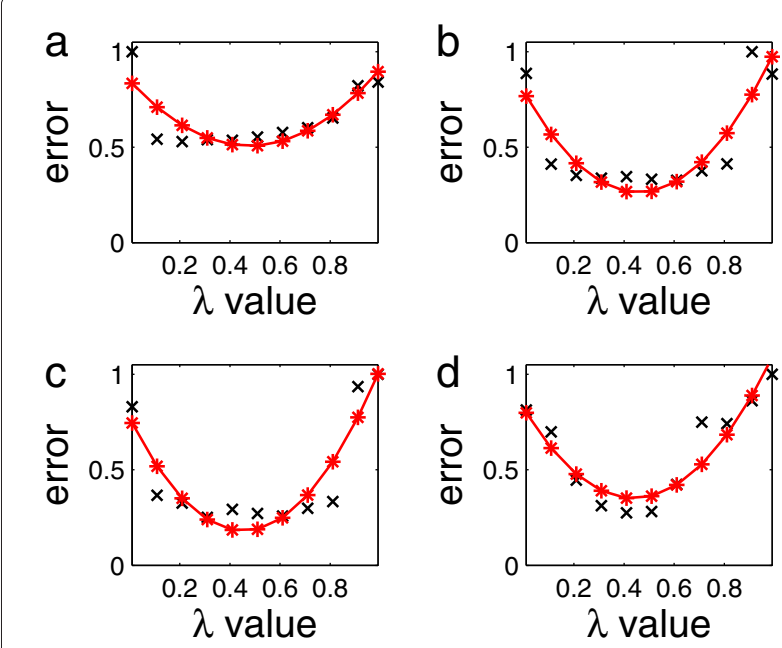

Figure 7 The normalized $\boldsymbol{e}(\boldsymbol{i})$ versus $\boldsymbol{\lambda}_{\boldsymbol{i}}$. (a) Errors vs. $\lambda$ at $\mathrm{SNR}=10 \mathrm{~dB}$. (b) Errors vs. $\lambda$ at SNR $=20 \mathrm{~dB}$. (c) Errors vs. $\lambda$ at SNR $=30 \mathrm{~dB}$. (d) Errors vs. $\lambda$ in noise-free case. phase (i.e., $\phi^{0}(\xi, \eta)=0$ ) and thresholding parameter $\lambda=0.5$ in simulations. Object plane measurement and Fourier plane measurement are contaminated by Gaussian noise with SNR $=30 \mathrm{~dB}$. The results of recovery using GS and GS-PhSparse are shown in Figure 8. Hereafter, truth magnitudes of wave field and corresponding truth phases are shown in Figure 3a,b, respectively. Exactly

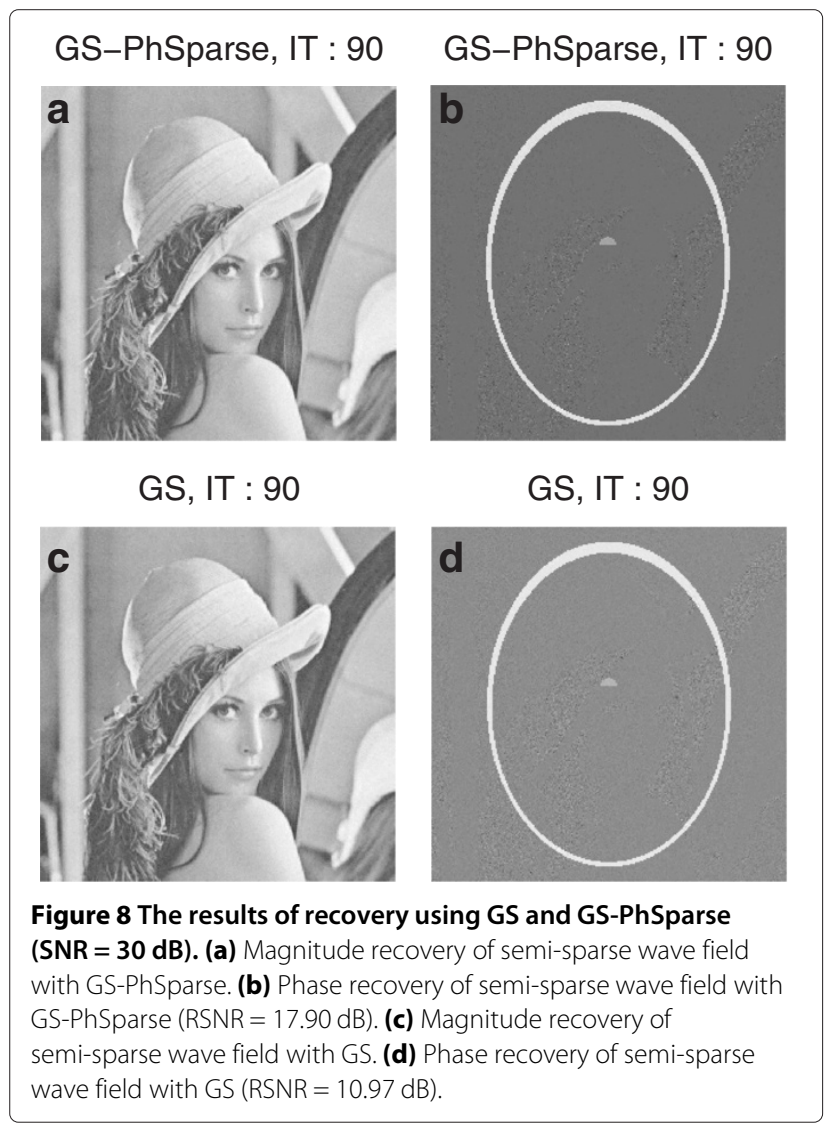




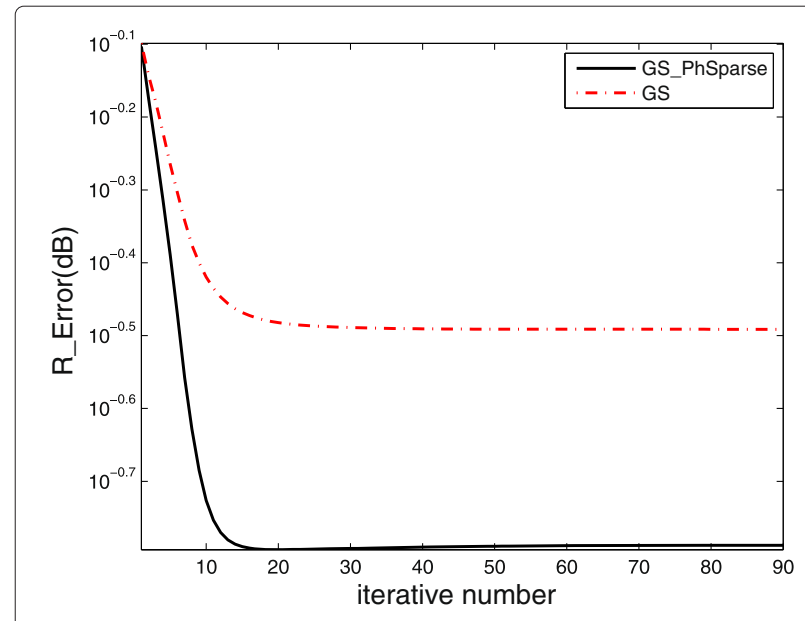

Figure 9 Normalized phase residual of semi-sparse wave field with GS and GS-PhSparse (SNR = $30 \mathrm{~dB}$ ).

speaking, magnitude recovery of semi-sparse wave field with GS-PhSparse approach is shown in Figure 8a, and the corresponding phase recovery is shown in Figure 8b. Magnitude recovery of the semi-sparse wave field with GS is shown in Figure 8c, and corresponding phase recovery is shown in Figure 8d. Meanwhile, we also calculate the values of RSNR which are 17.90 and $10.97 \mathrm{~dB}$ in Figure 8b,d, respectively, which show that the GS-PhSparse has higher reconstruction accuracy than GS. According to the results in Figure 8, the visual advantage of GS-PhSparse is obvious. Besides, normalized phase residuals of wave field with GS and GS-PhSparse are also simulated. The simulation result in Figure 9 shows that the GS-PhSparse approach has a faster convergence speed than the GS approach.

Meanwhile, we compare another two iterative projection approaches for phase retrieval: RAAR and RAARPhSparse. In the simulation, initialization of the phase and thresholding parameter $\lambda$ are the same as in GS and GSPhSparse. The results of recovery using RAAR and RAARPhSparse approaches are shown in Figure 10a,b,c,d. The values of RSNR are $17.96 \mathrm{~dB}$ in Figure 10b, and $11.13 \mathrm{~dB}$ in Figure 10d. According to the results of Figure 10, again, the visual advantage of the proposed approach is obvious. Moreover, normalized phase residuals of semi-sparse wave field with RAAR and RAAR-PhSparse are also calculated. The simulation result is shown in Figure 11. It shows that the RAAR-PhSparse approach also has faster residual convergence speed than the RAAR approach.

Besides, we also simulate the recovery performances of GS, GS-PhSparse, RAAR, and RAAR-PhSparse for SNR = $20 \mathrm{~dB}$. The results of recovery using GS and GS-PhSparse approaches are shown in Figure 12a,b,c,d, and the values of RSNR are $4.67 \mathrm{~dB}$ in Figure $12 \mathrm{~b}$ and $3.36 \mathrm{~dB}$ in

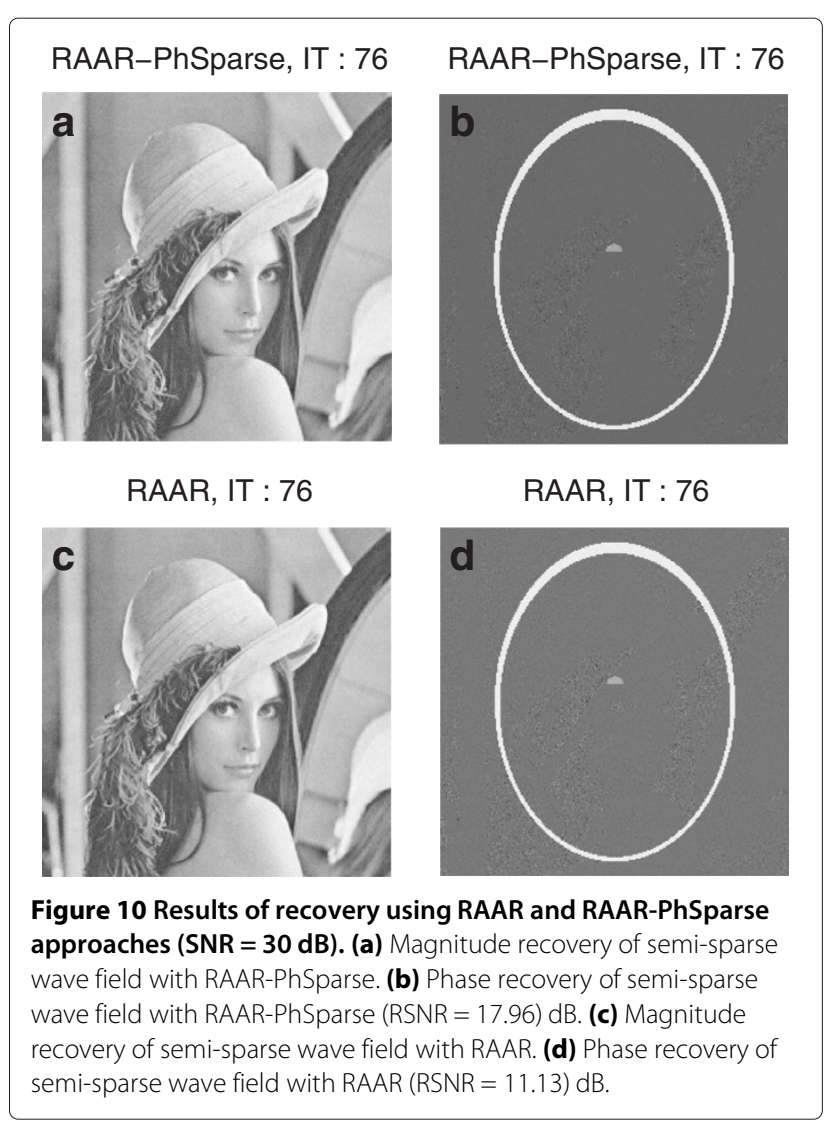

Figure 12d. The corresponding normalized phase residuals of semi-sparse wave field with GS and GS-PhSparse are plotted in Figure 13, which shows that the GS-PhSparse approach has smaller residual than the GS approach. The results of recovery using RAAR and RAAR-PhSparse approaches are shown in Figure 14a,b,c,d, and the values of RSNR are $5.31 \mathrm{~dB}$ in Figure $14 \mathrm{~b}$ and $3.51 \mathrm{~dB}$ in

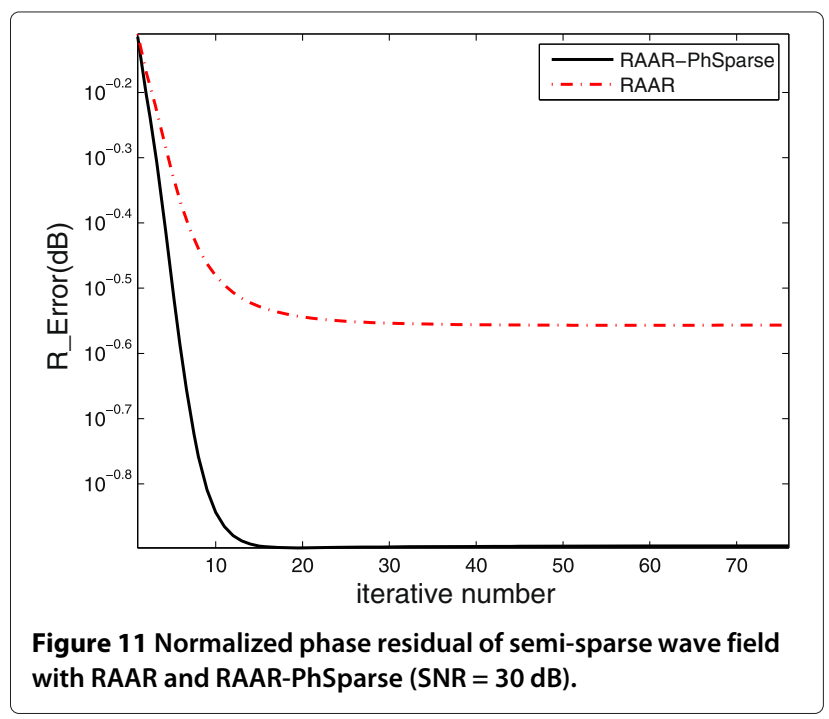




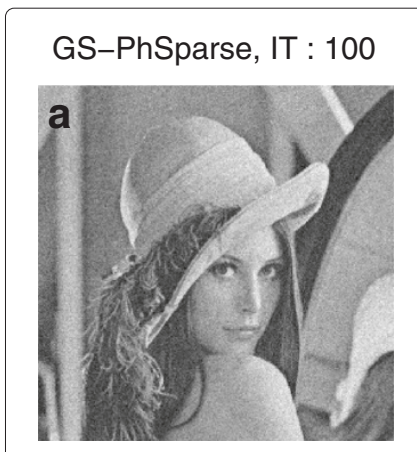

GS, IT : 100

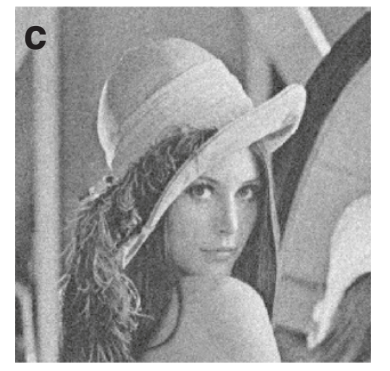

GS, IT : 100

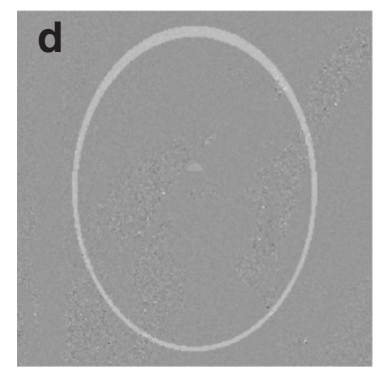

Figure 12 The results of recovery using GS and GS-PhSparse (SNR $=\mathbf{2 0} \mathrm{dB})$. (a) Magnitude recovery of semi-sparse wave field with GS-PhSparse. (b) Phase recovery of semi-sparse wave field with GS-PhSparse (RSNR $=4.67 \mathrm{~dB}$ ). (c) Magnitude recovery of semi-sparse wave field with GS. (d) Phase recovery of semi-sparse wave field with GS $(R S N R=3.36 \mathrm{~dB})$.

Figure 14d. The normalized phase residuals of the semisparse wave field with RAAR and RAAR-PhSparse are plotted in Figure 15. According to the results of Figures 12 to 15 , the visual advantages of the proposed approach are obvious.

Experiment 5. In Experiments 1, 2, and 4, we assume the same noise level for both object plane measurement

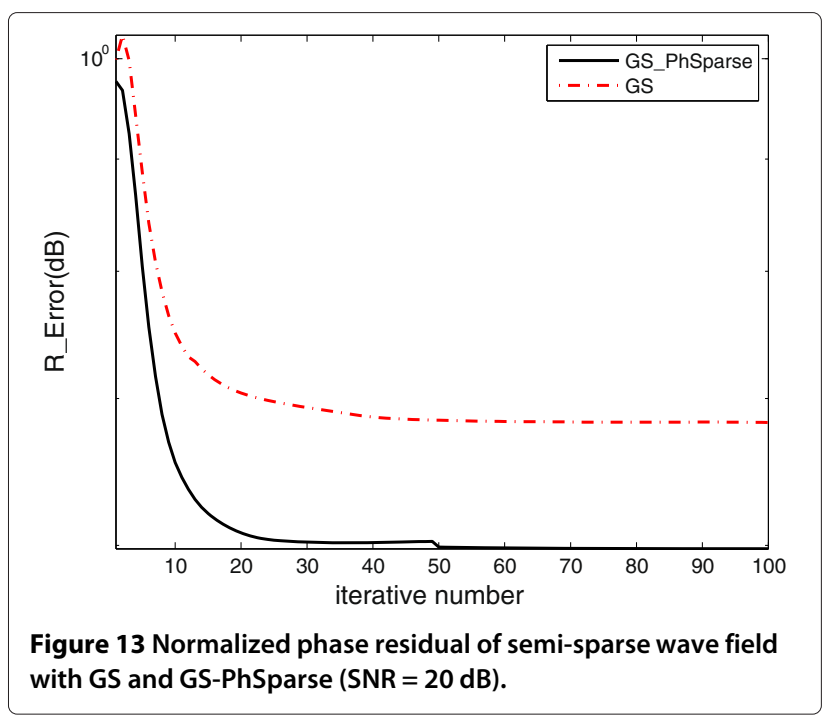

RAAR-PhSparse, IT : 100 RAAR-PhSparse, IT : 100

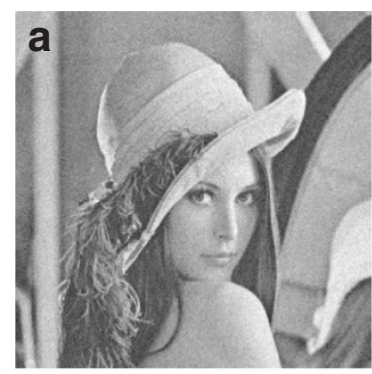

RAAR, IT : 100

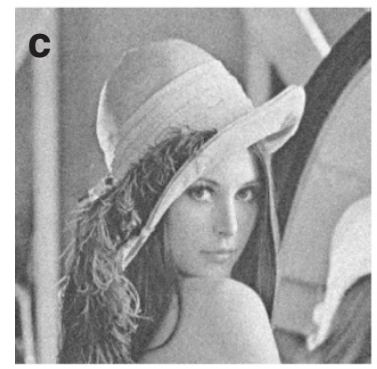

Figure 14 Results of recovery using RAAR and RAAR-PhSparse approaches ( $(\mathbf{N R R}=\mathbf{2 0} \mathrm{dB}$ ). (a) Magnitude recovery of semi-sparse wave field with RAAR-PhSparse. (b) Phase recovery of semi-sparse wave field with RAAR-PhSparse (RSNR = 5.31 dB). (c) Magnitude recovery of semi-sparse wave field with RAAR. (d) Phase recovery of semi-sparse wave field with RAAR (RSNR $=3.51 \mathrm{~dB})$. and Fourier plane measurement $(\mathrm{SNR}=30$ or $\mathrm{SNR}=$ $20 \mathrm{~dB}$ simultaneously). However, in this experiment, it shows that there are different effects when different noise levels are imposed on different measurements. Assuming two measurements, one is $\mathrm{SNR}=30 \mathrm{~dB}$, and the other is $\mathrm{SNR}=20 \mathrm{~dB}$. We calculate the RSNR of the recovered phases for different combinations of noise levels, such as (20 and $30 \mathrm{~dB}),(30$ and $20 \mathrm{~dB}),(30$ and $30 \mathrm{~dB})$, and $(20$

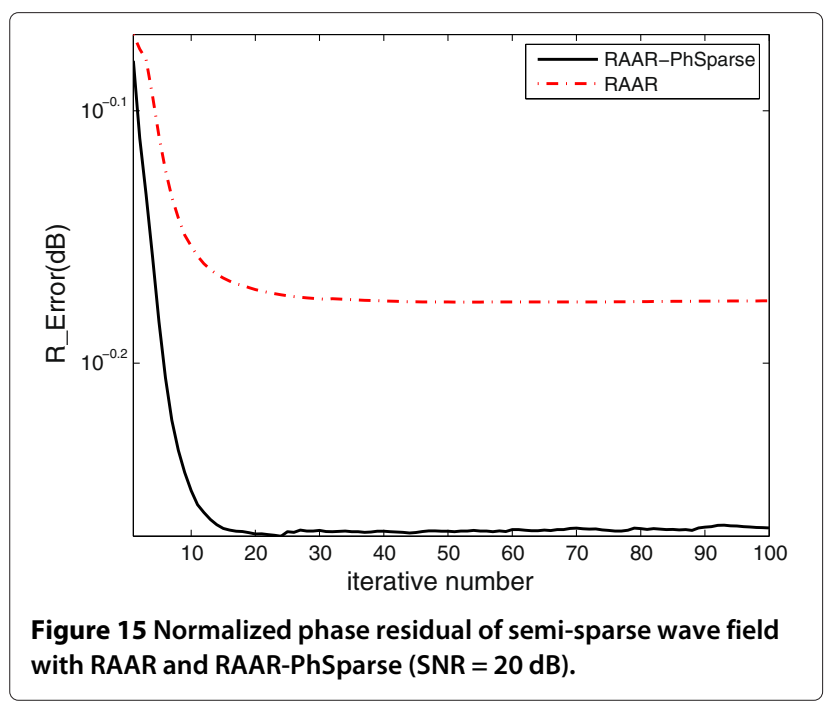


and $20 \mathrm{~dB}$ ), respectively. For example, (20 and $30 \mathrm{~dB})$, it means that the SNR on the object measurement plane is $20 \mathrm{~dB}$ and that on the Fourier measurement plane is $30 \mathrm{~dB}$. The other combinations have the same meaning, and the unit $\mathrm{dB}$ is ommitted for simplicity in Table 1. According to the results in Table 1, we find that different approaches have different sensitivities to different plane noises. GS and GS-PhSparse approaches are more sensitive to Fourier measurement plane noise level, but the RAAR and RAAR-PhSparse approaches are more sensitive to object measurement plane noise level. They have different robustness to noise.

Remark 4. Robust to noise: In the above experiments, Gaussian noise levels are with SNR $=30$ and $20 \mathrm{~dB}$. They are so small as to be worthy of formal discussion. As a matter of fact, the proposed approach is also suitable for larger noise level. In the case, we average multiple noisy measurements from object plane and Fourier measurement plane. We calculate the reconstruct performance of the proposed approach in SNR $=10 \mathrm{~dB}$, and we averaged 50 trial noisy measurements. The values of RSNR with GSPhSparse and GS are 11.61 and $7.51 \mathrm{~dB}$, respectively. And, the values of RSNR with RAAR-PhSparse and RAAR are 11.78 and $7.55 \mathrm{~dB}$, respectively. Therefore, the proposed approach with multiple measurements is suitable for lager noisy cases.

Experiment 6. We discuss how to deal with the largescale phase retrieval problem in the experiment. In the simulation, the wave field size is assumed be $1,024 \times$ 1,024 . The magnitude distribution and phase distribution of the semi-sparse wave field are shown in Figure 16a,d, respectively. All approaches terminate after a given number of iterations (30 iterations in RAAR-PhSparse and RAAR, 60 iterations in GS-PhSparse and GS). Object plane measurement and Fourier plane measurement are contaminated by Gaussian noise with $\mathrm{SNR}=30 \mathrm{~dB}$. The other parameters are the same as in Experiment 4. For large-scale phase retrieval problem, one popular solution is to divide wave field with $1,024 \times 1,024$ into several smaller wave fields (say 16 wave fields with $256 \times 256$ ). For each smaller wave field, we can use the same procedures in Experiment 4 to reconstruct each smaller wave

Table 1 Recovered RSNR (dB) for different SNR combinations

\begin{tabular}{lcccc}
\hline Approaches & $\mathbf{( 2 0 , 3 0 )}$ & $\mathbf{( 3 0 , 2 0 )}$ & $\mathbf{( 3 0 , 3 0 )}$ & $\mathbf{( 2 0 , 2 0 )}$ \\
\hline GS & 10.43 & 3.95 & 11.84 & 3.39 \\
GS-PhSparse & 14.86 & 5.83 & 17.08 & 4.84 \\
RAAR & 4.01 & 10.52 & 11.16 & 3.47 \\
RAAR-PhSparse & 5.83 & 15.44 & 17.89 & 5.14 \\
\hline
\end{tabular}

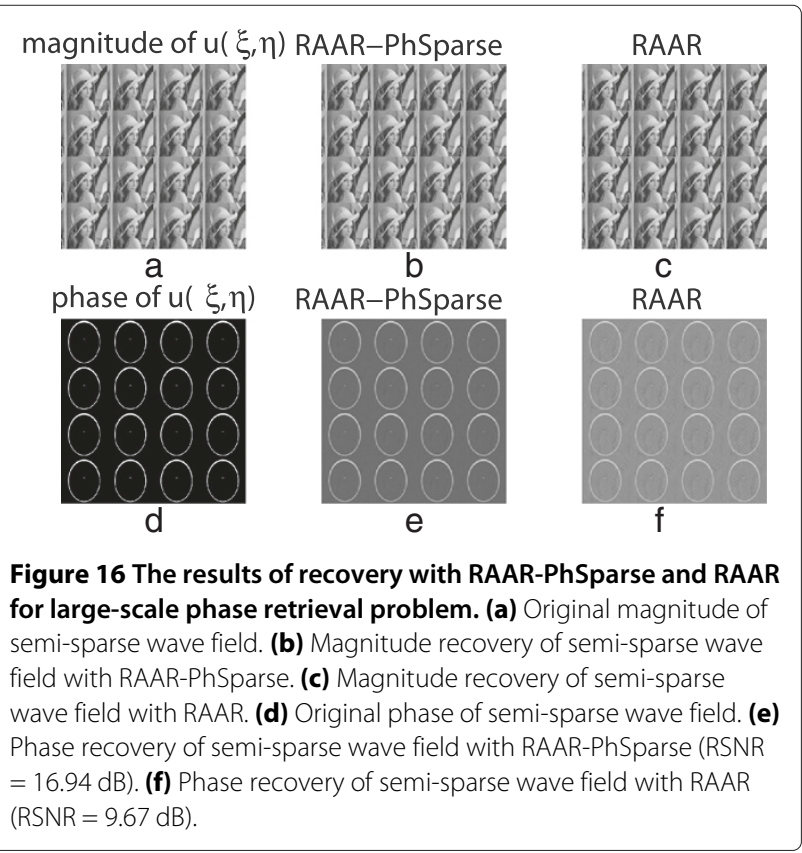

field. Once all the smaller wave fields with $256 \times 256$ are reconstructed, the wave field with $1,024 \times 1,024$ is determined.

However, considering the complexity of the iterative projection-type approach, for the wave field size of 1,024 $\times 1,024$, we need not divide it into several smaller wave fields in the experiment. A wave field with $1,024 \times$ 1,024 can be reconstructed with the proposed approach directly. The results of recovery with RAAR-PhSparse and

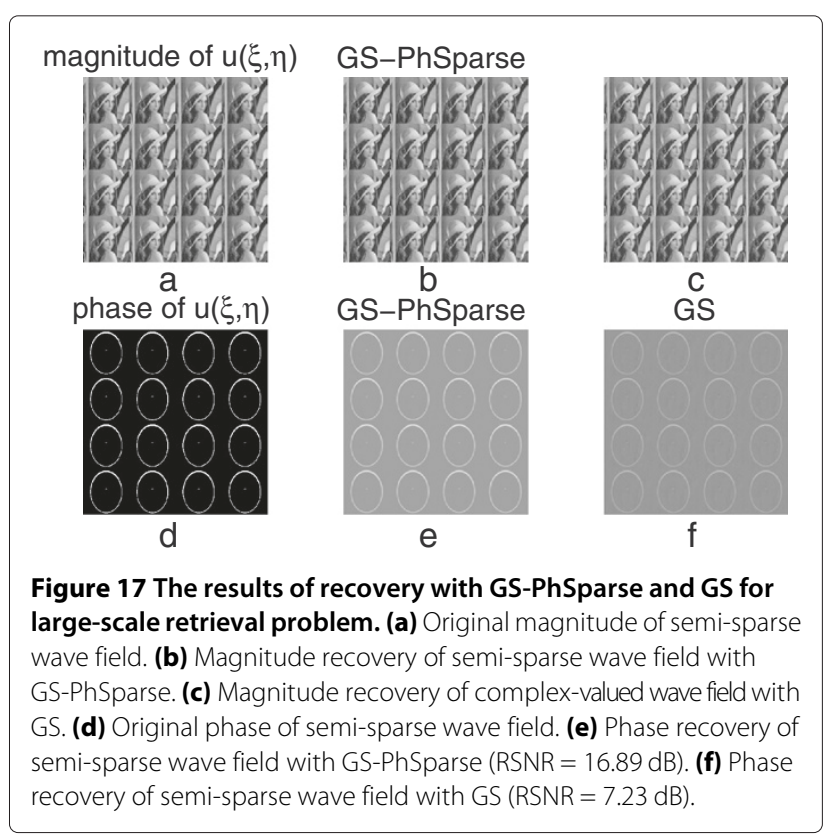


RAAR are shown in Figure 16. And, the results of recovery with GS-PhSparse and GS are shown in Figure 17. Exactly speaking, the magnitude recovery of semi-sparse wave field with RAAR-PhSparse approach is shown in Figure 16b, and corresponding phase recovery is shown in Figure 16e. The magnitude recovery of the semi-sparse wave field with RAAR is shown in Figure 16c, and corresponding phase recovery is shown in Figure 16f. The magnitude recovery of the semi-sparse wave field with the GS-PhSparse approach is shown in Figure 17b, and corresponding phase recovery is shown in Figure 17e. The magnitude recovery of the semi-sparse wave field with GS is shown in Figure 17c, and corresponding phase recovery is shown in Figure 17f.

Experiment 7. In the experiment, we verify the proposed approach which has the ability of enhancing the resolution. The magnitude distribution and phase distribution of wave field are the same as in Experiment 6, which are shown in Figure 18a,d, respectively. In simulation, both noise level and iterations are the same as those in Experiment 6, too. However, in order to illustrate the ability of enhancing the resolution in the proposed approach, only a half magnitude on the Fourier measurement plane was utilized in the simulation. The results of recovery using RAAR-PhSparse and RAAR are shown in Figure 18. And, the results of recovery using GS-PhSparse and GS are shown in Figure 19. From Figures 18 and 19, the visual advantages of the proposed approach are obvious. Meanwhile, we calculate the values of RSNR with RAAR-PhSparse and RAAR in Figure 18, which are 12.37

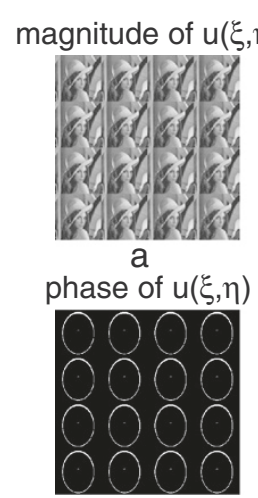

d

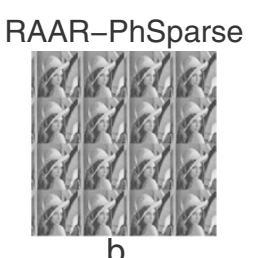

RAAR-PhSparse

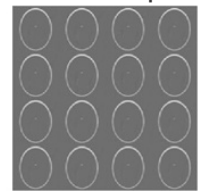

e
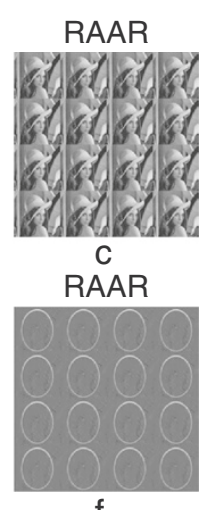

Figure 18 The results of recovery with RAAR-PhSparse and RAAR using partial magnitude measurement. (a) Original magnitude of semi-sparse wave field. (b) Magnitude recovery of semi-sparse wave field with RAAR-PhSparse. (c) Magnitude recovery of semi-sparse wave field with RAAR. (d) Original phase of semi-sparse wave field. (e) Phase recovery of semi-sparse wave field with RAAR-PhSparse (RSNR $=12.37 \mathrm{~dB}$ ). (f) Phase recovery of semi-sparse wave field with RAAR $($ RSNR $=1.31 \mathrm{~dB})$.

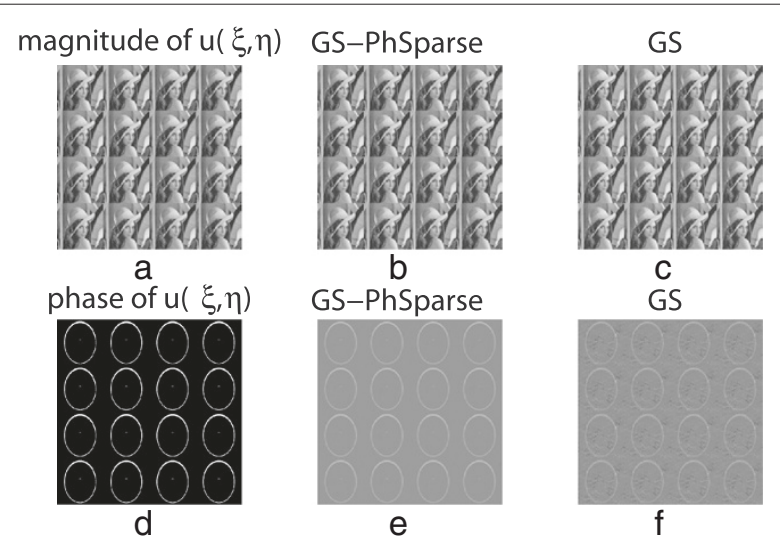

Figure 19 The results of recovery with GS-PhSparse and GS using partial magnitude measurement. (a) Original magnitude of semi-sparse wave field. (b) Magnitude recovery of semi-sparse wave field with GS-PhSparse. (c) Magnitude recovery of semi-sparse wave field with GS. (d) Original phase of semi-sparse wave field. (e) Phase recovery of semi-sparse wave field with GS-PhSparse (RSNR $=12.32$ dB). (f) Phase recovery of semi-sparse wave field with GS (RSNR $=1.19$ $d B)$.

and $1.31 \mathrm{~dB}$, respectively. The values of RSNR with GSPhSparse and GS in Figure 19, which are 12.32 and 1.19 $\mathrm{dB}$, respectively. Obviously, the proposed approach has the ability of enhancing the resolution.

A more detailed simulation comparison between the iterative projection approaches for phase retrieval and those with phase sparse constraint is not reported in this paper. As a matter of fact, all iterative projection approaches for phase retrieval with phase sparse constraint have a good recovery quantity and faster residual convergence speed than the counterparts.

\section{Conclusions}

The problem of 2D phase retrieval, namely, recovery of a 2D semi-sparse wave field from magnitudes of both wave field and its Fourier transform is addressed in the paper. The key aspect of our work is that the phase sparse constraint is applied in each iteration by retaining only phase elements that are larger than thresholding parameter and setting others equal to zero and thus refine the estimate of the phase. Unlike the standard phase retrieval approaches, this allows us to have variable phase support in each iteration in the literature. Simulation experiments demonstrate advantages of the proposed approach, including rapid convergence, small residual error, noise stability,s and suitability in large-scale phase retrieval problems.

\section{Competing interests}

The authors declare that they have no competing interests.

\section{Acknowledgements}

This paper is dedicated to welcome Shiwei Zou (nicknamed Xiao Bai Long) into this world and God bless her! The authors would like to thank the anonymous reviewers for their comments that helped improve the quality of this article. The support is partly provided by the National Natural Science 
Foundation of China (61172140). Yipeng Liu is supported by FWO PhD/postdoc grant: G.0108.11 (compressed sensing).

\section{Author details}

${ }^{1}$ School of Electronic Engineering, University of Electronic Science and Technology of China, No. 2006, Xiyuan Ave, West Hi-Tech Zone, Chengdu 611731, China. ${ }^{2}$ Air Control and Navigation Institution, Air Force Engineering University, Xi'an, 710000, China. ${ }^{3}$ ESAT-STADIUS/iMinds Future Health Department, Department of Electrical Engineering, KU Leuven, Leuven 3001, Belgium.

\section{Received: 30 September 2013 Accepted: 12 February 2014}

\section{Published: 28 February 2014}

\section{References}

1. J Dainty, JR Fienup, Phase retrieval and image reconstruction for astronomy. Image Recovery: Theory Appl. 13, $231-275$ (1987)

2. PMcManamon, An overview of optical phased array technology and status. Proc. SPIE. 5947, 59470I-10 (2005)

3. RA Gonsalves. Proc. SPIE. 21, 829-832 (1982)

4. D Dragoman, Applications of the Wigner distribution function in signal processing. EURASIP J. Adv. Sig. Proc. 10, 1520-1534 (2005)

5. Z Yang, Robust compressive phase retrieval via L1 minimization with application to image reconstruction (Cornell University, Ithaca, 2013). arXiv:1302.0081

6. V Davidoiu, B Sixou, M Langery, F Peyrin, Non-linear phase retrieval combined with iterative thresholding in wavelet coordinates. Paper presented at the 20th European signal processing conference (EUSIPCO, Bucharest, 27-31 Aug 2012), pp. 27-31

7. H Ohlsson, A Yang, R Dong, S Sastry, Compressive phase retrieval from squared output measurements via semidefinite programming (2011). arXiv:1111.6323

8. EJ Candes, XD Li, Solving quadratic equations via phaseLift when there are about as many equations as unknowns. Found. Comput. Math (2013). doi:10.1007/s10208-013-9162-z

9. I Waldspurger, A d'Aspremont, S Mallat, Phase recovery, maxcut and complex semidefinite programming (Cornell University, Ithaca, 2012). arXiv:1206.0102

10. K Jaganathan, S Oymak, B Hassibi, Phase retrieval for sparse signals using rank minimization. Paper presented at the IEEE international conference on acoustics, speech, and signal processing (ICASSP, Kyoto, 25-30 March 2012), pp. 3449-3452

11. K Jaganathan, S Oymak, B Hassibi, Sparse phase retrieval: convex algorithms and limitations (Cornell University, Ithaca, 2013). arXiv:1303.4128v1

12. RW Orengo, WO Saxton, A practical algorithm for the determination of phase from image and diffraction plane pictures. Optik. 35, 237-246 (1972)

13. JR Fienup, Phase retrieval algorithms: a comparison. Appl. Opt. 21, 2758-2769 (1982)

14. HH Bauschke, PL Combettes, DR Luke, Phase retrieval, error reduction algorithm, and Fienup variants: a view from convex optimization. J. Opt. Soc. Amer. A. 19, 1334-1345 (2002)

15. V Elser, Phase retrieval by iterated projections. J. Opt. Soc. Amer. A. 20, 40-55 (2003)

16. HH Bauschke, PL Combettes, DR Luke, Hybrid projection reflection method for phase retrieval. J. Opt. Soc. Amer. A. 20, 1025-1034 (2003)

17. DR Luke, Relaxed averaged alternating reflections for diffraction imaging. Inverse Probl. 21, 37-50 (2005)

18. S Marchesini, A unified evaluation of iterative projection algorithms for phase retrieval. Rev. Sci. Instrum. 78, 1-10 (2007)

19. YP Liu, MD Vos, I Gligorijevic, V Matic, YQ Li, SV Huffel, Multi-structural signal recovery for biomedical compressive sensing. IEEE Trans. Biomed. Eng. 60, 2794-2805 (2013)

20. G Gui, W Peng, F Adachi, Sub-Nyquist rate ADC sampling-based compressive channel estimation. Wireless Commun. Mobile Comput. 10 (2013). doi: $10.1002 /$ wcm 2372

21. G Gui, F Adachi, Improved least mean square algorithm with application to adaptive sparse channel estimation. EURASIP J. Wireless Commun. Netw (2013). doi:10.1186/1687- 1499-2013-204.3
22. F Marvasti, A Amini, F Haddadi, M Soltanolkotabi, BH Khalaj, A Aldroubi, S Sanei, J Chambers, A unified approach to sparse signal processing. EURASIP J. Adv. Sig. Proc (2012). doi:10.1186/1687-6180-2012-44

23. Y Shechtman, Y Eldar, A Szameit, M Segev, Sparsity based sub-wavelength imaging with partially incoherent light via quadratic compressed sensing. Opt. Express. 19, 14807-14822 (2011)

24. S Mukherjee, C Seelamantula, An iterative algorithm for phase retrieval with sparsity constraints: application to frequency domain optical coherence tomography. Paper presented at the IEEE international conference on acoustics, speech, and signal processing (ICASSP, Kyoto, 25-30 March 2012), pp. 553-556

25. Y Shechtman, A Beck, YC Eldar, GESPAR: efficient phase retrieval of sparse signals (Cornell University, Ithaca, 2013). arXiv:1301.1018

26. P Netrapalli, P Jain, Phase retrieval using alternating minimization (Cornell University, Ithaca, 2013). arXiv:1306.0160v1

27. V Katkovnik, J Astola, DR Luke, High-accuracy wave field reconstruction: decoupled inverse imaging with sparse modeling of phase and amplitude. J. Opt. Soc. Amer. A. 29, 44-54 (2012)

28. V Katkovnik, J Astola, DR Luke, Phase retrieval via spatial light modulator phase modulation in $4 \mathrm{f}$ optical setup: numerical inverse imaging with sparse regularization for phase and amplitude. J. Opt. Soc. Amer. A. 29, 105-116 (2012)

29. A Migukin, V Katkovnik, J Astola, Advanced multi-plane phase retrieval using graphic processing unit: augmented Lagrangian technique with sparse regularization. Proc. SPIE. 8429, 84291N (2012). doi:10.1117/12.922343

30. A Migukin, M Agour, V Katkovnik, Phase retrieval in 4 f optical system: background compensation and sparse regularization of object with binary amplitude. Appl. Opt. 52, A269-A280 (2013)

31. A Migukin, Iterative Phase Retrieval fromMultiple Noisy Observations: Variational and Sparse Object Approximation Techniques. PhD Thesis, Computer Science Department, Tampere University of Technology, 2012

doi:10.1186/1687-6180-2014-24

Cite this article as: Fan et al:: Iterative projection approach for phase retrieval of semi-sparse wave field. EURASIP Journal on Advances in Signal Processing 2014 2014:24.

\section{Submit your manuscript to a SpringerOpen ${ }^{\circ}$ journal and benefit from:}

- Convenient online submission

Rigorous peer review

- Immediate publication on acceptance

- Open access: articles freely available online

- High visibility within the field

- Retaining the copyright to your article

Submit your next manuscript at $\boldsymbol{\nabla}$ springeropen.com 